\title{
Diamonds for beam instrumentation
}

\author{
Erich Griesmayer $^{1}$ \\ CIVIDEC Instrumentation GmbH
}

Schottengasse 3A/1/41, A-1010 Vienna, Austria

E-mail: erich.griesmayer@cividec.at

\section{Pavel Kavrigin}

CIVIDEC Instrumentation $\mathrm{GmbH}$

Schottengasse 3A/1/41, A-1010 Vienna, Austria

E-mail: pavel.kavrigin@cividec.at

\begin{abstract}
Diamond is perhaps the most versatile, efficient and radiation tolerant material available for use in beam detectors with a correspondingly wide range of applications in beam instrumentation. Numerous practical applications have demonstrated and exploited the sensitivity of diamond to charged particles, photons and neutrons. In this paper, a brief description of a generic diamond detector is given and the interaction of the CVD diamond detector material with protons, electrons, photons and neutrons is presented. Latest results of the interaction of SCVD diamond with $14 \mathrm{MeV}$ mono-energetic neutrons are shown.
\end{abstract}

Technology and Instrumentation in Particle Physics 2014

2-6 June, 2014

Amsterdam, the Netherlands

1

Speaker 


\section{Introduction}

Diamond detectors are currently exciting a lot of attention. Their high radiation tolerance, their rapid response of the order of nanoseconds, and their low dark current of the order of picoamperes make them excellent particle detectors for applications in beam instrumentation devices [1]. It has long been known that diamonds fluoresce and generate electrical signals when irradiated. The emergence of single-crystal and poly-crystalline CVD diamond made it possible to provide detector material in precise shapes with uniform and predictable properties, while modern electronics make it possible to fully exploit the pico-second structure of the diamond response. These basic ingredients provide all that is needed for an extremely wide range of high-energy particle monitors and counters. The development of diamond detectors is a field where the basic physics is known, but technology is constantly pushing the boundaries outwards.

- Photons can be monitored above $5.5 \mathrm{eV}$, which corresponds to the conduction band, using a diode configuration in which the photo-excitation current is measured. In the range of $2 \mathrm{keV}$ up to $50 \mathrm{keV}$ diamond fluorescence monitors are widely used. Above $50 \mathrm{keV}$, the photoionized electrons ejected from the diamond start to create ionization in their own right and detectors configured as ionization chambers can be used, even for single-photon detection.

- For neutrons, a diamond monitor configured as an ionization chamber can be used with a converter from thermal energies up to $6 \mathrm{MeV}$ and above this energy there is no need for the converter. Recent tests at $n_{-}$TOF (CERN) have gone up to $1 \mathrm{GeV}$ neutrons [4].

- For electrons, diamond is used in the ionization mode and is frequently configured as a quadrant monitor in synchrotron light sources.

- Possibly the main advance in diamond detectors is currently in the detection of protons and ions. Giga-particle counting up to $3.5 \mathrm{TeV}$ has been demonstrated at the LHC (CERN) [1, 5 ] and carbon ions have been calorimetrically measured in the energy range of $30 \mathrm{MeV}$ at ISOLDE (CERN) [2].

\section{Principle of operation}

Figure 1 shows the operating principle of a diamond detector. Diamond is a semiconductive material. Particles traversing the detector liberate by ionization electrons and holes, which drift in the externally applied electric field and induce a current signal in a preamplifier. The ionization energy for CVD diamond is $13 \mathrm{eV} / \mathrm{eh}$-pair and the stopping power for minimum ionizing particles (MIP) is $490 \mathrm{eV} / \mu \mathrm{m}$ [6].

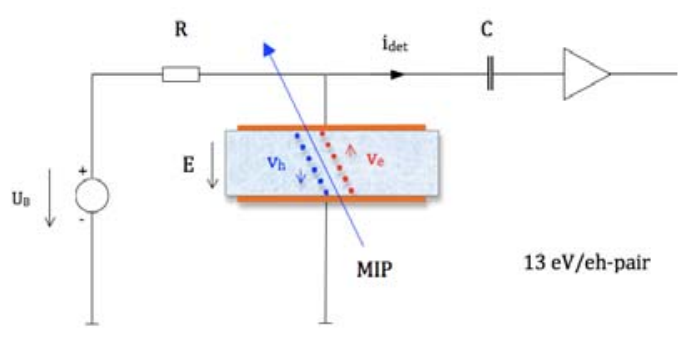

FIGURE 1. Principle of a diamond detector. The ionization energy of CVD diamond is $13 \mathrm{eV}$.

In a single crystalline detector with a thickness $d=500 \mu \mathrm{m}$, the drift time $t_{d}$ is of the order of $6 \mathrm{~ns}$ with an applied electric field of $1 \mathrm{~V} / \mu \mathrm{m}$. The corresponding drift velocity $v_{d}$ is $105 \mathrm{~m} / \mathrm{s}$ at a bias voltage $U_{B}=500 \mathrm{~V}$. The loading resistor $R=1 \mathrm{M} \Omega$ and the coupling capacitance $C=1 \mathrm{nF}$. 


\section{Types of operation}

\subsection{Proton interaction}

Protons ionize the CVD diamond on their passage through the material with an efficiency of essentially $100 \%$. The stopping power represents the energy loss of the particles in the detector in $\mathrm{eV} / \mu \mathrm{m}$. The function of energy loss versus particle energy corresponds to the BetheBloch function (see Figure 2). The stopping power shows a maximum at $100 \mathrm{keV}$ and a minimum at $3 \mathrm{GeV}$.

The values of $\mathrm{d} E / \mathrm{d} x$ can easily be converted into the deposited charge by division of the ionization energy of $13 \mathrm{eV} / \mathrm{eh}$ pair. Figure 3 shows the ionization charge in $\mathrm{fC}$ for the example of a $500 \mu \mathrm{m}$ thick detector.

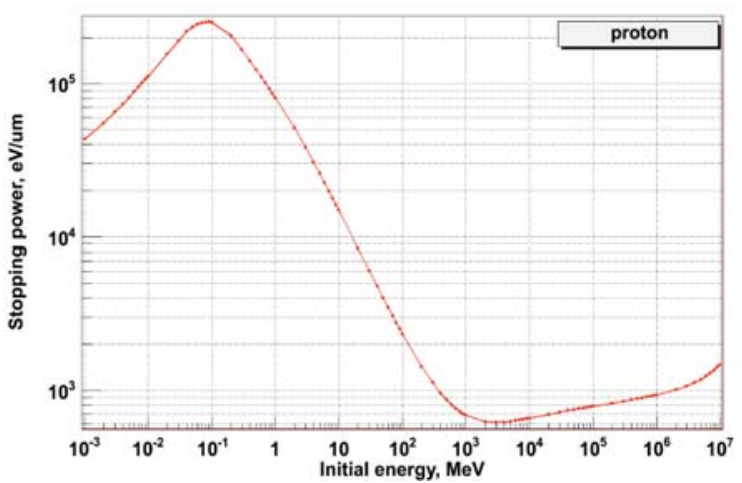

FIGURE 2. Bethe-Bloch function representing the stopping power of protons in CVD diamond.

For detecting single particles the level of ionization must be higher than the noise of the preamplifier. In Figure 3 the limit is drawn at $1 \mathrm{fC}$. Above this threshold, single particles can be detected. Below this threshold particle bunches are required to generate a signal that exceeds the threshold.

At energies below $100 \mathrm{keV}$ protons are absorbed and proton bunches are required in order to generate a detectable signal above $1 \mathrm{fC}$. At energies below $10 \mathrm{MeV}$ protons are absorbed (calorimetric mode) but they generate a signal above threshold, which makes single-particle detection possible. Above $10 \mathrm{MeV}$ protons traverse the detector (counting mode) and generate signals where single particle detection is possible.

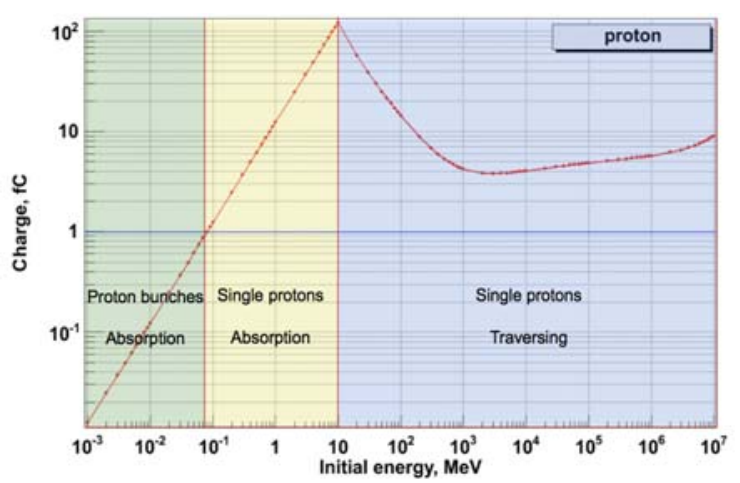

FIGURE 3. Ionization characteristics of protons in $500 \mu \mathrm{m} \mathrm{sCVD} \mathrm{diamond.}$ 
Diamond detectors were used for calorimetric and spectroscopic applications with $23 \mathrm{MeV}$ carbon ions at ISOLDE [2]. At LHC single-particle detection is currently applied in the Diamond Beam Loss Monitors for $3.5 \mathrm{TeV}$ protons [3]. In particular, the time resolution of $1 \mathrm{~ns}$ is interesting for this application, as the bunch separation at LHC is $25 \mathrm{~ns}$, making measurements of ghost bunches feasible. For medical applications with proton energies in the range of $70 \mathrm{MeV}$ to $250 \mathrm{MeV}$ diamond detectors are used for phase measurements and beam profile monitoring [5].

\subsection{Electron interaction}

The same approach can be applied to quantifying the electron interaction. Figure 4 shows the charge generation of electrons in a diamond detector.

Above $400 \mathrm{keV}$, electrons traverse the detector and are individually detectable (counting mode). Between $80 \mathrm{keV}$ and $400 \mathrm{keV}$, electrons will be absorbed by the detector, but they are still individually detectable (calorimetric mode). Below $80 \mathrm{keV}$ only electron bunches generating over $1 \mathrm{fC}$ can be resolved from the noise.

Diamond detectors are used in electron accelerators for synchrotron light sources as beam loss monitors where the sub-nanosecond time resolution is needed.

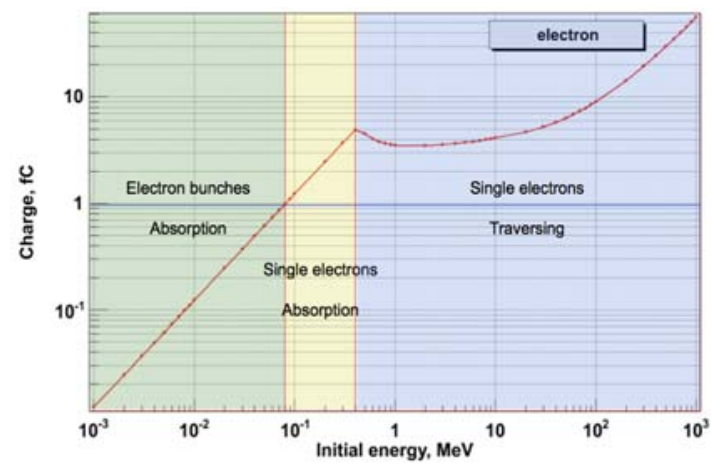

FIGURE 4. Ionization characteristics of electrons in $500 \mu \mathrm{m} \mathrm{sCVD} \mathrm{diamond.}$

\subsection{Photon interaction}

Figure 5 shows the three interaction processes in diamond in the range of $10 \mathrm{keV}$ to $1 \mathrm{TeV}$ that contribute to the ionization left by photons in the detector. As mentioned in the introduction, photons can also be detected at much lower energies (from $5.5 \mathrm{eV}$ using a diode configuration in which the photo-excitation current is measured and, in the range of $2 \mathrm{keV}$ up to $50 \mathrm{keV}$, using diamond fluorescence monitors). However, these low energy examples are not considered here.

- The photo-electric effect creates one primary electron per photon (with the full energy of the photon minus the binding energy of the electron) and this electron creates secondary ionization according to the graph in Figure 4.

- $\quad$ Scattered photons produce both a primary electron and a scattered photon that share the incident photon energy. The primary electron will create secondary ionization according to Figure 4 and the scattered photon can, in principle, participate in further scattering or the photoelectric effect. However, this potentially complicated situation is largely avoided because the low interaction rate for photons in diamond ensures that the majority of the scattered photons escape from a $500 \mu \mathrm{m}$ thick detector without further interaction. 


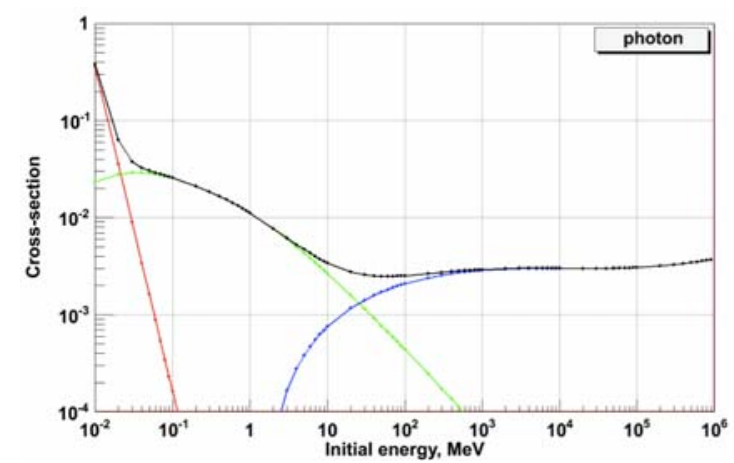

FIGURE 5. Cross section of photon interaction in $500 \mu \mathrm{m} \mathrm{sCVD} \mathrm{diamond.} \mathrm{The} \mathrm{photo-electric} \mathrm{effect} \mathrm{is}$ drawn in red, Compton scattering in green and pair production in blue. Total cross section is shown in black. The photo-electric effect dominates in the region up to $20 \mathrm{keV}$ where Compton scattering takes over. Pair production becomes important from $2 \mathrm{MeV}$ onwards and becomes the dominant process at $20 \mathrm{MeV}$. These three effects create secondary ionization.

- In pair production, the primary electron and positron share equally the energy of the incident photon minus the production energy of the pair $(1.022 \mathrm{MeV})$ and both particles create secondary ionization according to Figure 4.

Diamond detectors are used as dosimeters in medical X-ray applications [7]. For future applications in synchrotron light sources like XFEL, diamond detectors are under investigation as intensity monitors and to measure the position and the timing of photon bunches [8][9][10][11]. Diamond detectors are promising candidates for the X-ray plasma diagnostics in harsh irradiation conditions [12].

\subsection{Neutron interaction}

Diamond detectors were tested at the $n_{-}$TOF facility at CERN [4]. The interaction between neutrons and CVD diamond is shown in Figure 6 over an energy range from eV to $\mathrm{GeV}$ neutrons [13].

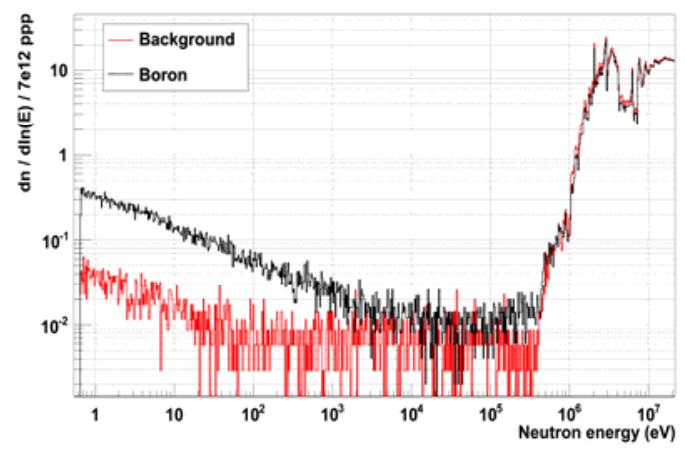

FIGURE 6. Measurement with a $500 \mu \mathrm{m}$ pCVD diamond in a neutron beam (red) and with a ${ }^{10} \mathrm{~B}$ converter foil (black).

Neutrons have a small interaction probability with matter. To increase this probability, especially in the energy range below $1 \mathrm{keV}$, converter materials such as ${ }^{10} \mathrm{~B}$ in the above example of Figure 6, can be used to increase the interaction rate (black curve). Those neutrons that pass through the converter foil without interaction still have a finite, but lower, chance of interaction in the main diamond detector. However, this signal may be qualified as background 
(red curve). For thermal neutrons with a ${ }^{10} \mathrm{~B}$ converter, the ${ }^{10} \mathrm{~B}(\mathrm{n}, \alpha){ }^{7} \mathrm{Li}$ reaction produces an $\alpha$-particle $(1.47 \mathrm{MeV})$ which is registered by the detector. Another commonly used converter is ${ }^{7} \mathrm{Li}$, where the ${ }^{7} \mathrm{Li}(\mathrm{n}, \mathrm{t})^{4} \mathrm{He}$ reaction produces a triton $(2.73 \mathrm{MeV})$ and an $\alpha$-particle $(2.05 \mathrm{MeV})$ which are both registered by the detector. At energies above $100 \mathrm{keV}$, neutrons can be measured through the $\mathrm{n} \rightarrow$ elastic reaction. Finally at neutron energies above $6 \mathrm{MeV}$, the $\mathrm{n} \rightarrow$ charged particle reactions are dominant.

Diamond detectors are used for spectroscopic applications in combination with highresolution digitizers. Neutron flux monitors are under investigation for nuclear fusion at ITER [14] for the detection of $14 \mathrm{MeV}$ neutrons. The use of a boron converter essentially increases the interaction probability of neutrons in the low-energy range below $1 \mathrm{keV}$. Diamond detectors can be used for a high-flux measurements at the neutron spallation sources [15], and in the plasma diagnostics via fast neutron measurements [16][17].

\subsection{Interaction of $14 \mathrm{MeV}$ neutrons and sCVD diamond}

Measurements with mono-energetic neutrons were taken at the Physikalisch Technische Bundesanstalt (PTB) Braunschweig using the $14 \mathrm{MeV}$ neutron beam at the Van der Graaf accelerator with sCVD diamond detectors [18]. Due to its fast response time and comparatively high radiation hardness, this material is a promising candidate for use in high radiation environments.

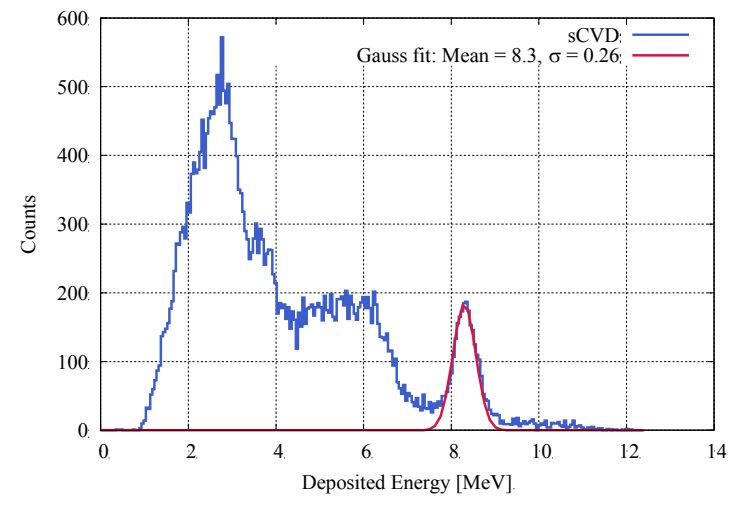

FIGURE 7. The neutron spectrum of the sCVD detector to $14 \mathrm{MeV}$ neutrons show the ${ }^{12} \mathrm{C}(\mathrm{n}, \alpha){ }^{9} \mathrm{Be}$ peak at $8.3 \mathrm{MeV}$ with an energy resolution of $260 \mathrm{keV}$.

Especially for neutron fluence monitoring at fusion reactors like ITER, this material should be explored for its neutron detection efficiency. The neutron spectrum of the SCVD detector can be seen in Figure 7. The peak corresponding to the ${ }^{12} \mathrm{C}(\mathrm{n}, \alpha){ }^{9} \mathrm{Be}$ is clearly visible at 8.3 MeV. The gaussian fit of this peak indicates a standard deviation for the energy resolution of $260 \mathrm{keV}$ for $14 \mathrm{MeV}$ neutrons.

\section{Conclusion}

Diamond detectors have a wide range of proven and potential applications as beam instrumentation devices. Charged particles, photons and neutrons can all be detected over a large energy range that is basically unlimited at the top end and limited by the noise in the detector at the lower end. The detection efficiency depends on the energy and in the case of charged particles it is close to $100 \%$. Photons and neutrons can be measured directly, but at lower energies the detection efficiency can be increased by the use of conversion foils. 


\section{Acknowledgements} article.

The authors would like to acknowledge the help of P.J. Bryant for his contributions to this

\section{References}

[1] B. Dehning, E. Effinger, D. Dobos, H. Pernegger, E. Griesmayer, Diamond Detectors as Beam Monitors, CERN-BE-2011-001 BI, March 2011.

[2] E. Griesmayer, H. Pernegger, D. Dobos, F. Wenander, J. Bergoz, H. Bayle, H. Frais-Kölbl, J. Leinweber, T. Aumeyr, High-Resolution Energy and Intensity Measurements with CVD Diamond at REX-ISOLDE, CERN BE-Note-2009-028.

[3] E. Griesmayer, B. Dehning, E. Effinger, D. Dobos, H. Pernegger, LHC Diamond Beam Loss Monitors, CERN ATS/Note/2011/048, June 2011.

[4] C. Rubbia et al., CERN/LHC/98-02 (EET).

[5] E. Griesmayer, B. Dehning, E. Effinger, H. Pernegger, D. Dobos, H. Frais-Kölbl, Test of a Diamond Detector Using Unbunched Beam Halo Particles, CERN-ATS-2010-027.

[6] W. Adam, C. Bauer, E. Berdermann et al., Development of CVD Diamond Radiation Detectors, RD42 Collaboration, 5th International Symposium on Diamond Materials, 1997.

[7] H. Frais-Kölbl, E. Griesmayer, T. Schreiner, D. Georg, H. Pernegger, Medical Beam Monitor - Preclinical Evaluation and Future Applications, NIM A (581), 2007.

[8] H. Aoyagi, et al., Beam Halo Monitor Using Diamond Detectors for XFEL/SPring-8, 11th European Particle Accelerator Conference, THPC146, June 2008.

[9] M. Di Fraia et al., X-Ray Beam Position Monitor Based on a Single Crystal Diamond Performing Bunch by Bunch Detection, J. Phys.: Conf. Ser. (425) 212001, 2013.

[10] K. Desjardins, D. Duran, S. Hustache, M. Pomorski, W. Shepard, Characterisation of CVD diamond devices as XPBMs at SOLEIL, J. Phys.: Conf. Ser. (425) 212004, 2013.

[11] J. Bohon, E. Muller, J. Smedley, Development of Diamond-based X-ray Detection for High-Flux Beamline Diagnostics, J. Synchrotron Radiation (17), 2010.

[12] B. Caiffi et al., Analysis of the Response of CVD Diamond Detectors for UV and sX-Ray Plasma Diagnostics Installed at JET, Nuclear Instruments and Methods (00) 1-4, 2014.

[13] Ch. Weiss, n_TOF Collaboration, private communication, CERN, September 2011.

[14] http://www.iter.org/newsline/272/1624

[15] M. Rebai et al., Diamond Detectors for Fast Neutron Measurements at Pulsed Spallation Sources, JINST 7 C05015, 2012.

[16] D. Lattanzi, M. Angelone, M. Pillon et al., Single Crystal CVD Diamonds as Neutron Detectors at JET, Fusion Engineering and Design (84) 7-11, 2009. 
[17] C. Cazzaniga et al., Single Crystal Diamond Detector Measurements of Deuterium-Deuterium and Deuterium-Tritium Neutrons in Joint European Torus Fusion Plasmas, Rev. Sci. Instrum. (85) 043506, 2014.

[18] C. Weiss et al., Response of CVD Diamond Detectors to 14 MeV Neutrons, CERN-ATS-Note-2012093 TECH, 2012. 